\title{
A HIERARCHICAL COUPLED MULTI-SCALE MODEL FOR SHORT FIBER COMPOSITES
}

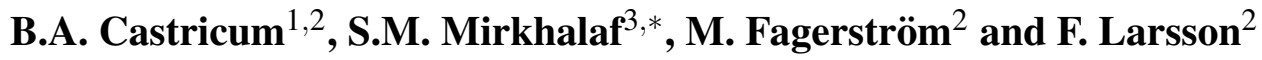 \\ ${ }^{1}$ Department of Mechanical Engineering, Eindhoven University of Technology, Eindhoven, \\ Netherlands \\ ${ }^{2}$ Division of Material and Computational Mechanics, Department of Industrial and Materials Science, \\ Chalmers University of Technology, Gothenburg, Sweden \\ ${ }^{3}$ Department of Physics, University of Gothenburg, Gothenburg, Sweden \\ * Corresponding author, mohsen.mirkhalaf@ physics.gu.se
}

Key words: Short fiber composites, Mechanical behaviour, Coupled multi-scale modelling, Orientation averaging

\begin{abstract}
Short Fiber Reinforced Composites (SFRCs) are being increasingly used in a variety of applications due to their interesting mechanical properties and ease of processing. For SFRCs, different micro-structural parameters (in addition to the constitutive behaviour of the matrix and reinforcement fibers), such as fiber orientation distribution, fiber aspect ratio and fiber/matrix interface strength play important roles in the macroscopic mechanical behaviour. Hence, to have an accurate and reliable modelling approach, using multi-scale models is a natural choice. In this study, a coupled multi-scale model is proposed using a recently developed micromechanical model and the Finite Element Method. The proposed model enables analysis of macroscopic specimens considering micro-structural properties.
\end{abstract}

\section{INTRODUCTION}

Short Fiber Reinforced Composites (SFRCs) are becoming more widely used for parts where traditionally plastic materials are used [1]. SFRCs are cost-effective and their ease of processing makes them favorable for products that require strength and lightweight.

To use SFRCs efficiently, the mechanical behaviour of these materials should be predicted quantitatively. The orientations of the fibers have a dominant influence on the macroscopic behaviour of these materials [2]. The orientations are largely influenced by the fabrication process, as shown in [3, 4]. Different fiber orientations will result in different mechanical responses. To fully model SFRCs in practical applications, a multi-scale model should be developed wherein each point of the part could potentially have different micro-structural characteristics.

Mean-field homogenization methods (such as Mori-Tanaka method) can be used to predict the mechanical response of SFRCs (see e.g. [5]). Also, using computational homogenization with realistic Representative Volume Elements (RVEs) has gained popularity for these materials [6, 7]. Computational homogenization is an accurate method to determine the material behaviour. However, the simulations are computationally expensive and, more importantly, the RVE generation can be challenging for SFRCs 
$[8,9]$. An alternative approach to the computational homogenization method is using the orientation averaging method [10]. An originally developed micromechanical model [11] is further developed by Mirkhalaf et al [12]. Finite Element simulations, performed on a Unit Cell, are used to calibrate the material properties for the extended model. In this study, a coupled multi-scale model is developed using the extended micromechanical model and Finite Element Method, by incorporating the micromechanical model in an FE package. Multi-scale simulations are conducted and the results are analyzed.

\section{MICROMECHANICAL MODEL}

This section describes the micromechanical model which is used to determine the material behaviour of a material point (integration point in FE context) based on micro-structural parameters. The micromechanical model is based on the orientation averaging approach proposed by Advani and Tucker [10]. This approach calculates the mechanical behaviour of a Unit Cell (single fiber embedded in matrix material) and then, based on the fiber orientations, the overall behaviour of the SFRC is calculated. The original micromechanical model is developed by Mirkhalaf et al. [11] and further extended in [12]. A two-step homogenization method is used to determine the material behaviour of SFRCs. In Figure 1, a schematic representation of this method is shown. In the first homogenization step, a Unit Cell (UC) is introduced which contains one fiber and the mechanical response of this UC is obtained via Finite Element Analysis. In this model, we use an elasto-plastic model for the matrix material. This modeling approach could be extended to use an elasto-viscoplastic model for a polymeric matrix material (e.g. $[13,14,15])$. In the second homogenization step, the homogenized properties of the composite are obtained using the UC homogenized properties

via orientation averaging. is accurate as shown in Element Analysis has to against the initial Finite the need for more FE simı
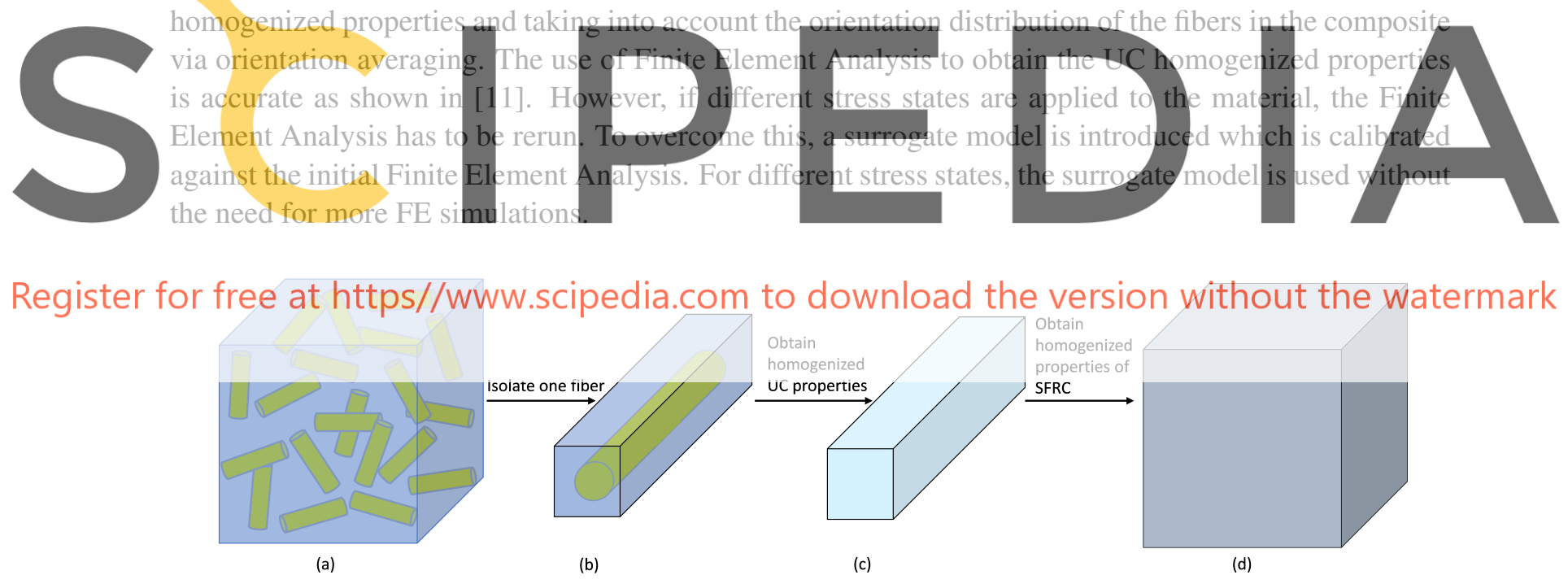

Figure 1: The two-step homogenization approach, (a): Composite with fibers, (b) to (c): First homogenization step, (c) to (d): Second homogenization step.

A rotation tensor $\mathbf{R}$ is introduced which maps the global configuration to the local configuration (denoted with superscript $L$ ) such that:

$$
\mathbf{e}_{i}^{L}=\mathbf{R} \cdot \mathbf{e}_{i} .
$$


The orientation of the fiber can be expressed as a vector $\mathbf{p}$ with two angles ( $\varphi$ and $\theta$ ), as schematically shown in Figure 2.

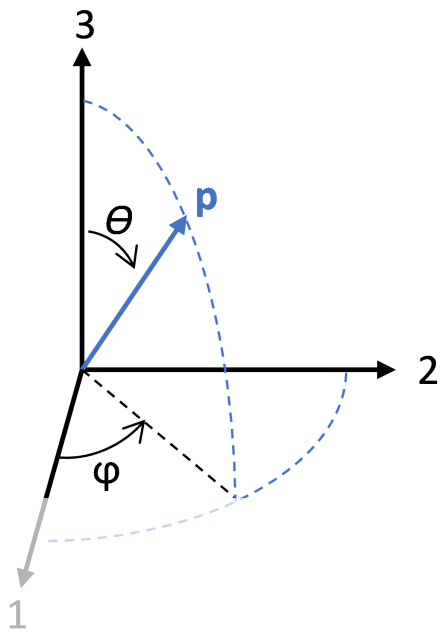

Figure 2: Spherical coordinate system to describe the orientation of the fiber in 3D space.

The rate of the composite stress and the UC stress are related by the following
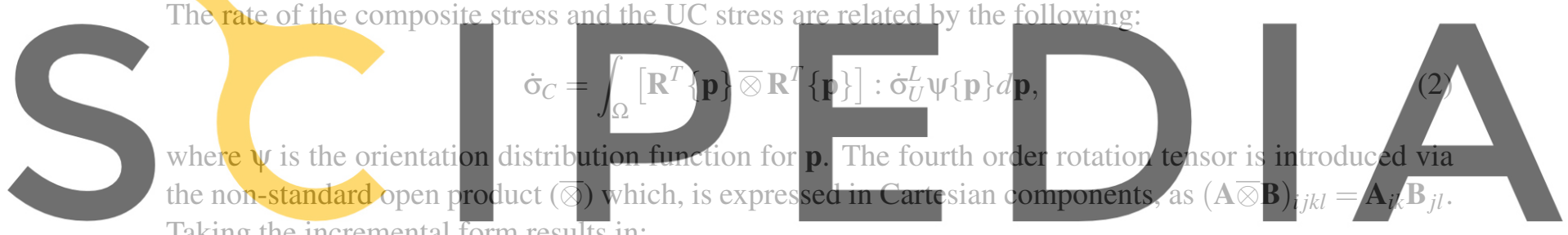

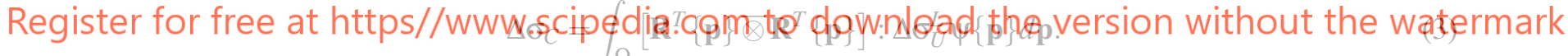

The Voigt interaction assumes that all UCs experience the same strain at the global configuration, and this strain is equal to the macroscopic (composite) strain. Thus, the incremental UC strain at the local configuration is obtained as:

$$
\Delta \varepsilon_{U}^{L}=[\mathbf{R}\{\mathbf{p}\} \bar{\otimes} \mathbf{R}\{\mathbf{p}\}]: \Delta \varepsilon_{C} .
$$

Now that the mechanical behaviour on the composite level is determined, the UC mechanical behaviour is required. A surrogate model is used to describe the UC deformation behaviour. The surrogate model is calibrated against the material properties obtained via Finite Element Analysis. A transversely isotropic material model is used where the elastic stiffness of the UC is expressed as:

$$
\mathbb{C}_{U}^{e}=\mathbb{C}_{U}^{e, i s o}+\left(C_{1}-1\right)\left(K+\frac{4 G}{3}\right)(\mathbf{A} \otimes \mathbf{A})
$$

where $\mathbb{C}_{U}^{e, i s o}$ is the standard isotropic elastic stiffness tensor and $\mathbf{A}$ is a tensor indicating the fiber orientation in the UC. $K$ and $G$ are the bulk and shear modulus, respectively. The parameter $C_{1}$ characterizes 
the transverse isotropic elasticity.

To capture nonlinear material behaviour, a transverse isotropic version of Hill's yield criteria is used, which is given by:

$$
\begin{aligned}
\Phi=\frac{1}{\left(1-C_{2}\right) \sigma_{\mathrm{y} 0}^{2}}[ & \left.C_{2}\left(\sigma_{U, 11}^{L}-\sigma_{U, 22}^{L}\right)^{2}+\left(\sigma_{U, 11}^{L}-\sigma_{U, 33}^{L}\right)^{2}+\left(\sigma_{U, 22}^{L}-\sigma_{U, 33}^{L}\right)^{2}\right] \\
& +\frac{2\left(2 C_{2}+1\right)}{\left(C_{2}+1\right) \sigma_{\mathrm{y} 0}^{2}}\left[\left(\sigma_{U, 12}^{L}\right)^{2}+\left(\sigma_{U, 32}^{L}\right)^{2}+\left(\sigma_{U, 23}^{L}\right)^{2}\right]-\alpha\left\{\bar{\varepsilon}^{p}\right\}
\end{aligned}
$$

In this equation, $\sigma_{y 0}$ is the initial yield stress and $C_{2}$ is the transversely isotropic yield parameter. $\alpha\left\{\bar{\varepsilon}^{p}\right\}$ is the isotropic hardening function, which is dependent on the effective plastic strain. In this work, the following isotropic hardening function is used:

$$
\alpha\left\{\bar{\varepsilon}^{p}\right\}=1+C_{3} \bar{\varepsilon}^{p}+C_{4}\left(\bar{\varepsilon}^{p}\right)^{2}+C_{5}\left(\bar{\varepsilon}^{p}\right)^{3} .
$$

The parameters $C_{3}, C_{4}$ and $C_{5}$ describe the isotropic hardening. In order to use the isotropic hardening, a definition of the effective plastic strain rate is required. The definition of the plastic strain rate used in this model is:

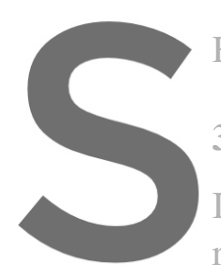

Here, $\lambda$ is the plastic multiplier.

3 COUPLED MULTI-SCALI

In a coupled multi-scale model, the required. The macro-scale problem

$$
\dot{\bar{\varepsilon}}^{p}=\frac{2 \lambda}{\sigma_{y 0}} .
$$

[16]. The constitutive behaviour in this multi-scale model is determined via the micro-scale problem

Register fopsfrieed at pretips/4:www.scipedia.com to download the version without the watermark

The macroscopic problem is obtained by the spatial discretization of the macroscopic equilibrium equa-

tion. The equilibrium needs to be solved for each (pseudo-) time increment such that the nodal displace-

ment vector $\mathbf{u}$ satisfies the balance:

$$
\mathbf{r}\{\mathbf{u}\}=\mathbf{f}_{\text {int }}\{\mathbf{u}\}-\mathbf{f}_{\text {ext }}=0 .
$$

After linearization of this equation, the macro-scale problem is solved. The macroscopic problem is coupled to the micromechanical model, where in each macroscopic point (each integration point in the FE context), the constitutive behaviour is determined by the micromechanical model. The FEM framework supplies the micromechanical model with the macroscopic strain in that integration point. The micromechanical model calculates the microscopic strain for each fiber (UC). The amount of microscopic strain (UC strain) is determined using the macroscopic strain (strain at the composite point) and based on the Voigt interaction. Once the microscopic strain is known for each UC, the UC stress is calculated. The micromechanical model calculates the homogenized stress and the tangent stiffness and returns them to the macroscopic problem. Figure 3 shows a schematic representation of the coupled multi-scale model. 


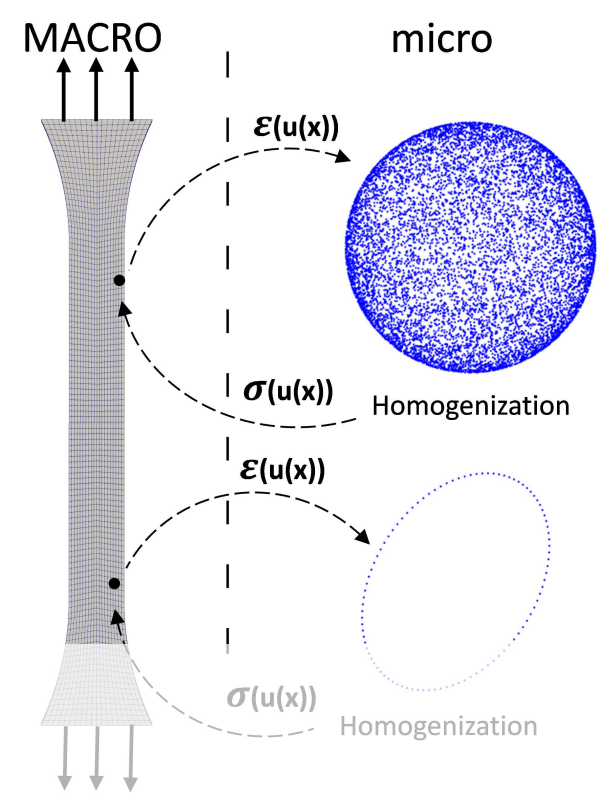

Figure 3: Schematic representation of the coupled multi-scale model. In each integration point, different microstructural characteristics (such as fiber orientation) can be used to obtain the mechanical behaviour.

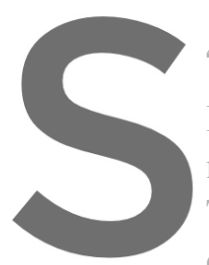

\section{RESULTS}

In this section, first, the

numerical simulation of a

To verify the coupled nuu
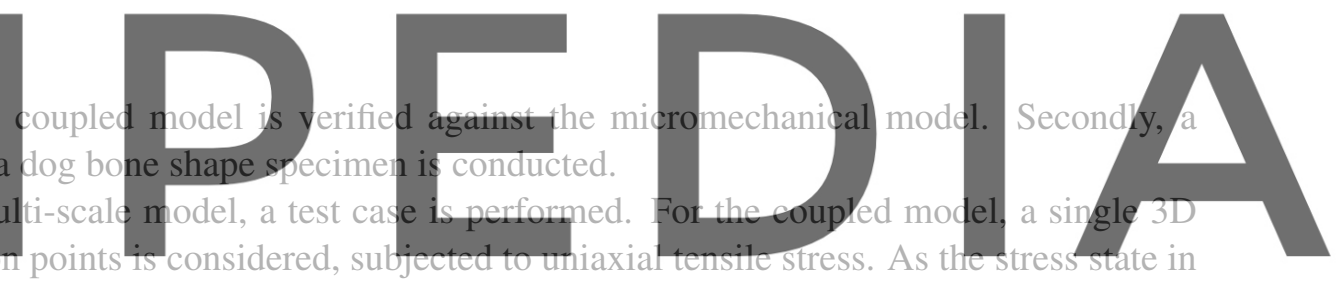

the element is uniform, this should result in the same stress state as the micromechanical model subjected

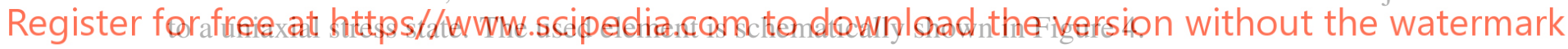

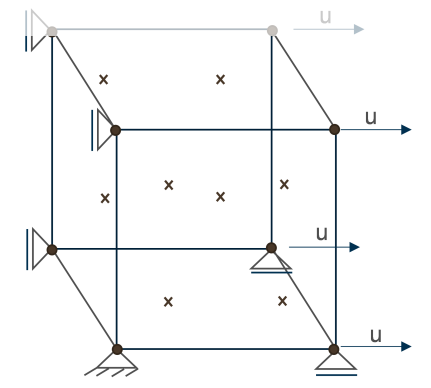

Figure 4: Representation of a single 3D element with 8 integration points and prescribed displacements and boundary conditions satisfying uniaxial stress.

A polypropylene matrix with short flax fibers is used as material. The fibers have a volume fraction of 0.13 , a fiber length of $1200 \mu \mathrm{m}$ and an aspect ratio of 75 . The orientation distribution of the fibers is 
isotropic. For micromechanical simulations, it is typically needed to obtain the required size (or number of orientations) for the micro-structural sample [17]. In these micromechanical simulations, 5000 random orientations are used, and the Voigt interaction is assumed. For the coupled simulations, the exact same orientations and interaction assumption is used. Figure 5 shows that the same stress-strain curves are obtained from the micromechanical and coupled models, as expected.
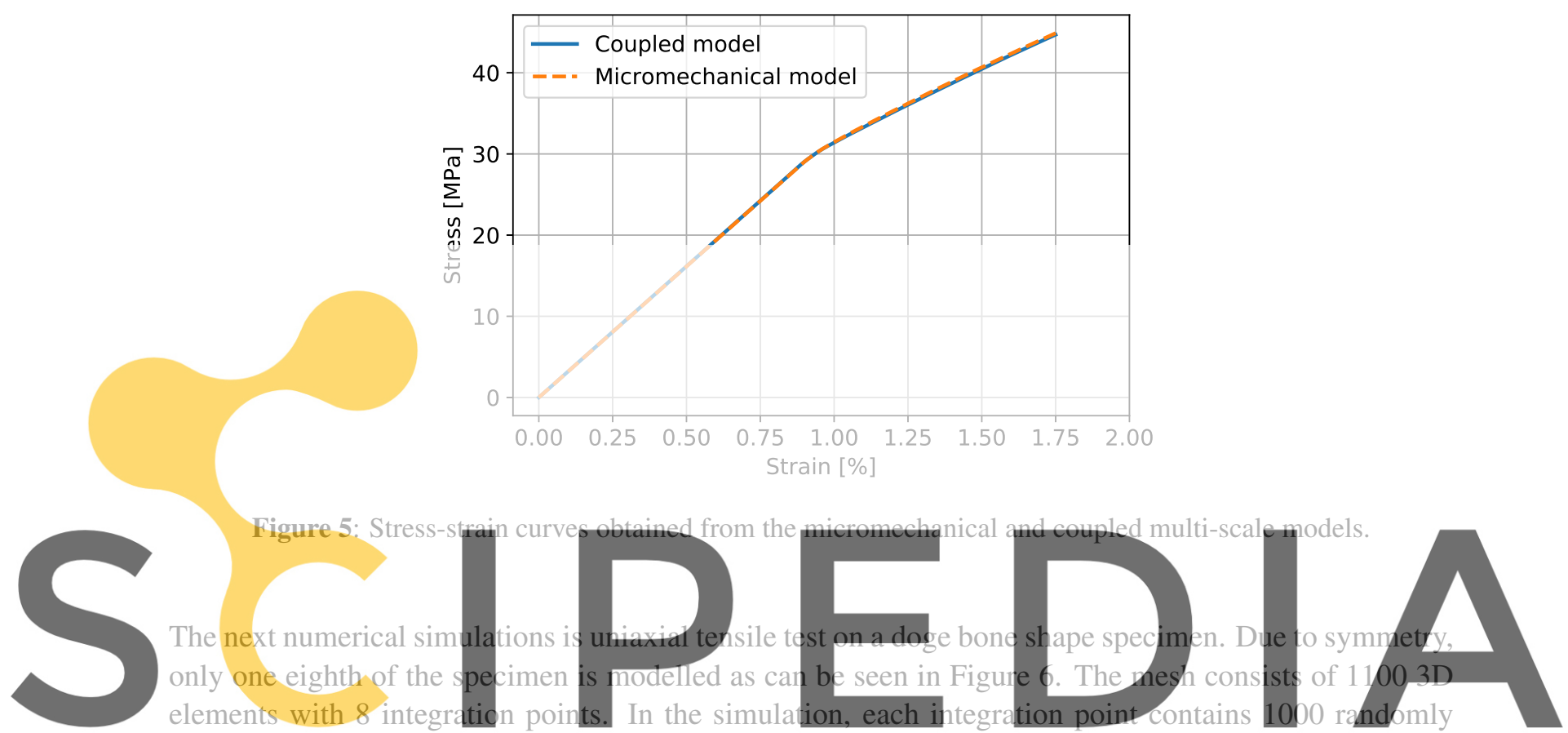
orientated fibers.

Register for free at https//www.scipedia.com to download the version without the watermark

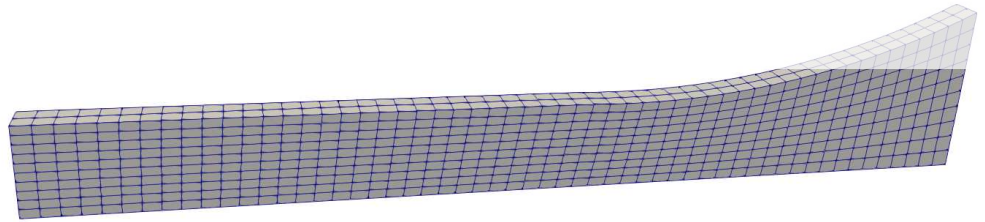

Figure 6: Finite Element mesh of one eighth of a dog-bone specimen used for the numerical study.

The displacement in $\mathrm{x}$-direction is shown in Figure 7 and the resulting stress in the specimen is shown in Figure 8. The results show that the multi-scale model is capable of non-linear material predictions wherein each integration point micro-structural parameters can be described. 


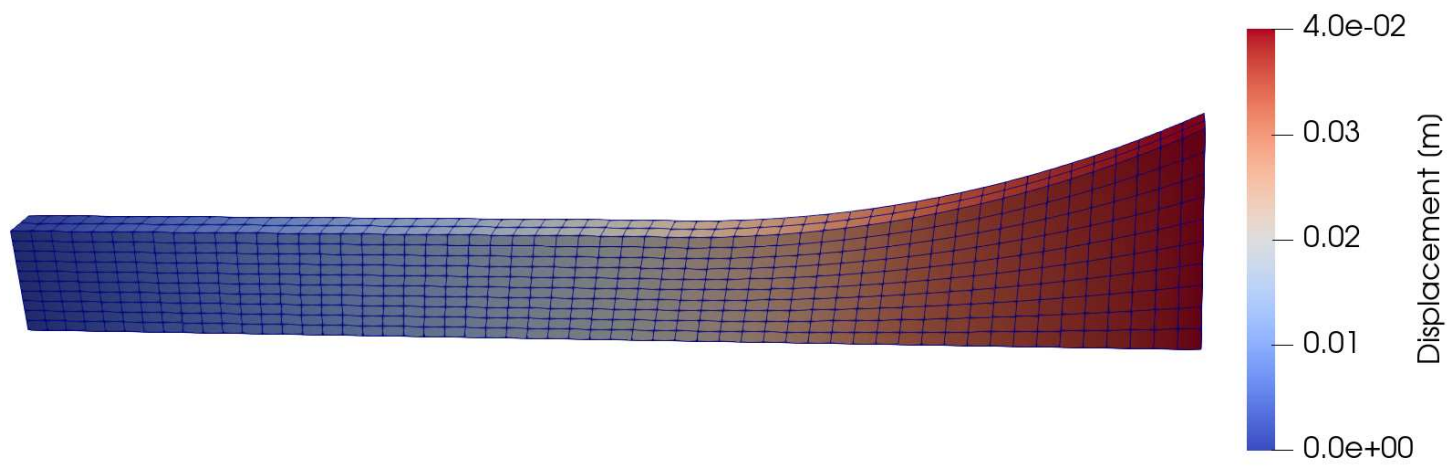

Figure 7: Final displacement of the sample in x-direction.

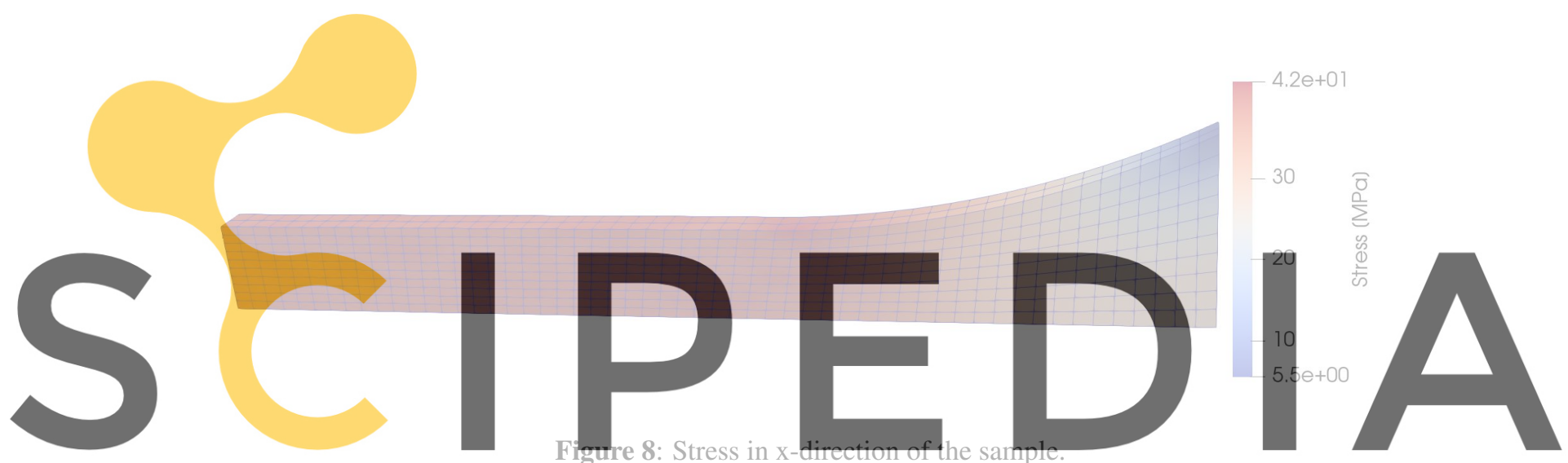

Register for free at https//www.scipedia.com to download the version without the watermark 5 CONCLUSIONS

In this study, a coupled multi-scale model is developed for non-linear elasto-plastic behaviour of short fiber reinforced composites. Two length scales, namely macroscopic and microscopic scales are considered in the model. For solving the equilibrium problem at the macro-scale, the Finite Element Method is used. For the microscopic problem, a micromechanical model, which was developed before, is used. The developed model is capable of simulating completely coupled macro-micro simulations, where the mechanical response of a macroscopic specimen is obtained considering micro-structural characteristics of all macroscopic points. This model provides an opportunity for simulating real life components which have different micro-structural properties at different parts of the structure. Since coupled analysis is performed with this model, simulations are time consuming, and improvements in terms of computational efficiency are needed.

\section{ACKNOWLEDGMENT}

B.A. Castricum acknowledge financial support from Erasmus+ programme of the European Union. S.M. Mirkhalaf gratefully acknowledges financial support from the Swedish Research Council (VR grant: 
2019-04715) and the University of Gothenburg. M. Fagerström is thankful for the support through Vinnova's strategic innovation programme LIGHTer, and from Area of Advance, Materials Science at Chalmers University of Technology.

\section{REFERENCES}

[1] M. Ramesh, K. Palanikumar, and K. H. Reddy. Plant fibre based bio-composites: Sustainable and renewable green materials. Renewable and Sustainable Energy Reviews, 79:558-584, 2017.

[2] W. Ogierman and G. Kokot. A study on fiber orientation influence on the mechanical response of a short fiber composite structure. Acta Mech, 227:173-183, 2016.

[3] J. Köbler, M. Schneider, F. Ospald, H. Andrä, and R. Müller. Fiber orientation interpolation for the multiscale analysis of short fiber reinforced composite parts. Computational Mechanics, 61:729$750,2018$.

[4] M. Zaidani, M. A. Omar, and S. Kumar. Coupling of injection molding process to mechanical properties of short fiber composites: A through process modeling approach. Journal of Reinforced Plastics and Composites, 34(23):1963-1978, 2015.

[5] S. Kammoun, I. Doghri, L. Adam, G. Robert, and L. Delannay. First pseudo-grain failure model for inelastic composites with misaligned short fibers. Composites Part A: Applied Science and Manufacturing, 42(12):1892-1902, 122011.

[6] S.M. Mirkhalaf, E.H. Eggels, A.T. Anantharange, F. Larsson, and M. Fagerström. Short fiber com-
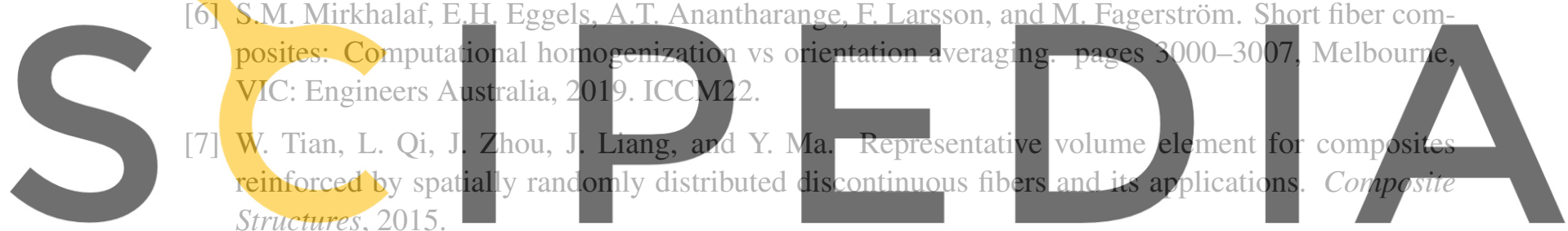

[8] S. Bargmann, B. Klusemann, J. Markmann, J.E. Schnapel, K. Schneider, C. Soyarslan, and

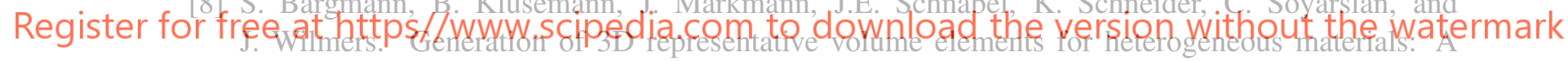
review, 72018.

[9] Y. Pan, L. Iorga, and A.A. Pelegri. Numerical generation of a random chopped fiber composite RVE and its elastic properties. Composites Science and Technology, 68(13):2792-2798, 102008.

[10] S. G. Advani and C. L. Tucker. The use of tensors to describe and predict fiber orientation in short fiber composites. Journal of Rheology, 31(8):751-784, 111987.

[11] S. M. Mirkhalaf, E. H. Eggels, T. J.H. van Beurden, F. Larsson, and M. Fagerström. A finite element based orientation averaging method for predicting elastic properties of short fiber reinforced composites. Composites Part B: Engineering, 202, 122020.

[12] S.M. Mirkhalaf, T.J.H. van Beurden, M. Ekh, F. Larsson, and M. Fagerström. A finite element based orientation averaging model for predicting elasto-plastic behaviour of short fiber reinforced composites (under review). 2021.

[13] S. M. Mirkhalaf, F. M. Andrade Pires, and R. Simoes. An elasto-viscoplastic constitutive model for polymers at finite strains: Formulation and computational aspects. Computers and Structures, 
166:60-74, 42016.

[14] S. M. Mirkhalaf, F. M. Andrade Pires, and R. Simoes. Modelling of the post yield response of amorphous polymers under different stress states. International Journal of Plasticity, 88:159-187, 12017.

[15] M. Mirkhalaf, J. A. W. van Dommelen, L. E. Govaert, J. Furmanski, and M. G. D. Geers. Micromechanical modeling of anisotropic behavior of oriented semicrystalline polymers. Journal of Polymer Science Part B: Polymer Physics, 57(7):378-391, 42019.

[16] R. de Borst, M. A. Crisfield, J. J. C. Remmers, and C.V. Verhoosel. Non-linear Finite Element Analysis. John Wiley \& Sons, Ltd, 72012.

[17] S. M. Mirkhalaf, F. M. Andrade Pires, and R. Simoes. Determination of the size of the Representative Volume Element (RVE) for the simulation of heterogeneous polymers at finite strains. Finite Elements in Analysis and Design, 119:30-44, 102016.
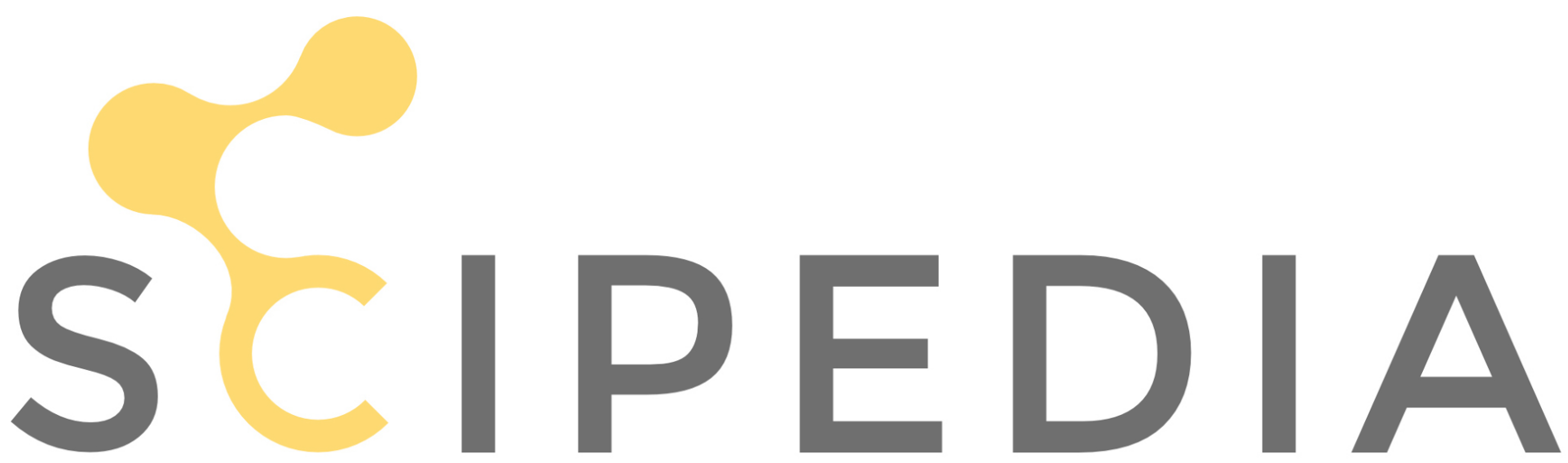

Register for free at https//www.scipedia.com to download the version without the watermark 